\title{
SINTOMATOLOGIA E MODIFICAÇÕES NO COTIDIANO DAS MULHERES NO PERÍODO DO CLIMATÉRIO
}

Rosiane Pitombeira' ${ }^{1}$, Francisca Elisângela Teixeira Lima² ${ }^{2}$ Fernanda Jorge Magalhães ${ }^{3}$, Ires Lopes Custódio4, Shérida Karanini Paz de Oliveira ${ }^{4}$

RESUMO: O objetivo do estudo foi averiguar as mudanças ocorridas na vida de mulheres durante o climatério e verificar os principais sintomas por elas referidos. A pesquisa foi realizada em unidade básica de saúde do Município de Fortaleza, Ceará e a amostra constituiu-se de 101 mulheres no período do climatério. A coleta de dados foi realizada por meio de entrevista, cujos resultados foram tabulados e organizados em quadros. Constatou-se que a sintomatologia associada à síndrome do climatério foi altamente prevalente, sendo que $92,07 \%$ das mulheres experimentaram pelo menos um dos sintomas, com maior frequência de fogachos, irritabilidade e esquecimento; entre as principais alterações no cotidiano, os sintomas relacionados à depressão e às alterações metabólicas foram os mais referidos, confirmando-se que a transição do climatério tem grande impacto na vida. O enfermeiro pode atuar junto às mulheres como facilitador para o enfrentamento e significação desta fase da vida. PALAVRAS-CHAVE: Climatério; Menopausa; Enfermagem em saúde comunitária; Cuidados de enfermagem.

\section{SYMPTOMATOLOGY AND MODIFICATIONS IN THE DAILY ROUTINES OF PREMENOPAUSAL WOMEN}

\begin{abstract}
The objective of this study was to investigate the changes which happen in women's lives during the premenopause and to check the principle symptoms they refer to. The research was carried out in a Basic Health Unit in the city of Fortaleza in the state of Ceará and the study population consisted of 101 premenopausal women. Data collection was via interview, and the results were analyzed and organized into tables. It was ascertained that the symptomatology associated with premenopause syndrome was highly prevalent, with $92.07 \%$ of the women experiencing at least one of the symptoms, most commonly hot flushes, irritability and forgetfulness; respecting the principle alterations in daily life, symptoms linked to depression and to metabolic changes were most referred to, confirming that the premenopausal transition has a large impact on life. The nurse can act with women as a facilitator for confronting and coming to terms with this phase of life.

KEYWORDS: Premenopause; Menopause; Nursing in community health; Nursing care.

\section{SINTOMATOLOGÍA Y CAMBIOS EN EL COTIDIANO DE LAS MUJERES EN EL PERÍODO DEL CLIMATERIO}

RESUMEN: El objetivo del estudio fue averiguar los cambios ocurridos en la vida de mujeres durante el climaterio y verificar los principales síntomas por ellas mencionados. La investigación fue realizada en unidad básica de salud del Municipio de Fortaleza, Ceará y la muestra se constituyó de 101 mujeres en el período del climaterio. Los datos fueron recogidos por medio de entrevista, cuyos resultados fueron tabulados y organizados en cuadros. Se ha constatado que la sintomatología asociada a la síndrome del climaterio fue alta, siendo que $92,07 \%$ de las mujeres tuvieron por lo menos uno de los síntomas, con mayor frecuencia de calores, irritabilidad y olvido; entre las principales alteraciones en el cotidiano, los síntomas asociados a la depresión y a las alteraciones metabólicas fueron los más mencionados, confirmándose que la transición del climaterio trae gran impacto en la vida. El enfermero puede actuar como facilitador para el afrontamiento y significación de esta fase de la vida. PALABRAS-CLAVE: Climaterio; Menopausia; Enfermería en salud comunitaria; Cuidados de enfermería.

${ }^{1}$ Enfermeira da Universidade de Fortaleza - UNIFOR.

${ }^{2}$ Enfermeira. Doutora. Professora do Departamento e do Programa de Pós-Graduação em Enfermagem da Faculdade de Farmácia, Odontologia e Enfermagem da Universidade Federal do Ceará - UFC. Coordenadora do Grupo de Estudos sobre a Consulta de Enfermagem - GECE.

${ }^{3}$ Enfermeira. Especialista em Neonatologia. Mestranda em Enfermagem na Promoção da Saúde pelo Programa de Pós-Graduação em Enfermagem da Faculdade de Farmácia, Odontologia e Enfermagem da UFC. Membro do GECE.

${ }^{4}$ Enfermeira. Mestranda em Enfermagem na Promoção da Saúde pelo Programa de Pós-Graduação em Enfermagem da Faculdade de Farmácia, Odontologia e Enfermagem da UFC. Membro do GECE. 


\section{INTRODUÇÃO}

O envelhecimento populacional é uma realidade demográfica brasileira. Como consequência, esperase, nos próximos anos, um aumento progressivo na procura dos serviços de saúde, inclusive por mulheres com queixas relacionadas ao climatério. Reconhece-se que o climatério é influenciado por fatores biológicos, psicossociais e culturais, sendo necessário que o enfermeiro conheça esses fatores para prestar uma assistência mais qualificada, humanizada e eficaz ${ }^{(1)}$.

Entre as fases do ciclo vital feminino, encontra-se o climatério, caracterizado como a passagem da fase reprodutiva para a não reprodutiva, ajustando a mulher às alterações hormonais e emocionais. A menopausa, nomenclatura mais difundida, consiste na interrupção permanente da menstruação, após decorrer 12 meses de amenorréia, a qual ocorre em função da perda da atividade folicular ovariana, sendo um momento pontual do climatério ${ }^{(2)}$.

É considerado um fenômeno fisiológico de esgotamento dos folículos ovarianos seguido da queda progressiva da secreção de estradiol, culminando com a interrupção definitiva dos ciclos menstruais e o surgimento de sintomas característicos ${ }^{(3)}$.

Iniciando-se por volta dos 35 anos de idade, e alcançando o seu término aproximadamente aos 65 anos, o climatério atinge as mulheres causando-lhes sinais e sintomas que trazem desconfortos em menor ou maior grau, variando de mulher para mulher ${ }^{(2)}$. Entretanto, estudiosos ${ }^{(4)}$ afirmam que o diagnóstico de climatério pode ser feito pelo quadro clínico, já a confirmação do diagnóstico necessita da realização de exames laboratoriais, para se verificar os níveis de hormônio folículo estimulante, hormônio luteinizante, os quais se encontram elevados, e de estradiol, que se encontra diminuído.

Os sinais e sintomas típicos do período do climatério incluem fogachos, insônia, irritabilidade, depressão, sudorese, palpitação, cefaléia, esquecimento, problemas urinários, estresse, além de transtornos como: desajustes conjugais, problemas familiares; e também alterações na sexualidade, dentre outros ${ }^{(5)}$.

Considera-se, ainda, que a relação da mulher, neste período, com o próprio corpo e com o desejo sexual é marcada por diversos fatores. A depleção hormonal, a história de vida pessoal e familiar, as experiências afetivas, o espaço social que a mulher ocupa, a etnia, a raça, a classe social e o momento contemporâneo são alguns aspectos indissociáveis que constituem a experiência subjetiva feminina ${ }^{(6)}$.

Segundo projeções da Organização Mundial da Saúde, entre 1990 e 2025, o número de idosos aumentará entre sete e oito vezes em vários países da América Latina, África e Ásia. O Brasil, à semelhança de outros países latino-americanos, passa por um processo de envelhecimento populacional acelerado e intenso. Entre 1980 e 2000, a proporção de brasileiros com mais de 60 anos aumentou de $6,1 \%$ para $8,6 \%$, devendo chegar a $14 \%$ até 2025 , o que representará uma das maiores populações de idosos no mundo. As implicações sociais e concernentes ao envelhecimento populacional no âmbito da saúde pública são preocupantes, pois, ao contrário dos países mais desenvolvidos, onde o aumento da expectativa de vida foi gradual e aliado a um maior desenvolvimento tecnológico e científico, o mesmo não ocorreu nos países em desenvolvimento ${ }^{(7-8)}$.

A partir de então, percebe-se a importância de conhecer a sintomatologia das mulheres no período do climatério; como ocorre o início dessa fase; a evolução dos diversos sintomas; as mudanças de sua rotina e surgimentos de outros transtornos, possibilitando a atuação do enfermeiro como educador em saúde, permitindo, assim, prestar um atendimento de qualidade a essas mulheres, auxiliando-as no enfrentamento dessa fase.

Estudos epidemiológicos evidenciam que as mulheres que têm acesso às informações acerca do climatério vivenciam melhor essa fase. Nesse contexto, o enfermeiro atuante na Estratégia de Saúde da Família possui papel fundamental na formação de grupos de mulheres no climatério, a fim de proporcionar espaço propício para manifestações, trocas e reflexões sobre aspectos relevantes da experiência da mulher ${ }^{(9)}$.

Assim, surgiram os questionamentos: Que modificações são evidenciadas pela mulher nessa fase da vida? Qual a sintomatologia e os transtornos ocorridos na vida da mulher durante o climatério?

Têm-se como objetivos: averiguar as mudanças ocorridas na vida de mulheres durante o período do climatério e verificar os principais sintomas referidos pelas mulheres no período do climatério.

\section{METODOLOGIA}

Trata-se de um estudo descritivo, quantitativo, realizado na sala de espera de um ambulatório de ginecologia e clínica médica, existente em unidade básica de saúde, constando de uma unidade escola, localizado 
em Fortaleza-CE. A população foi composta por todas as mulheres atendidas no referido ambulatório, as quais estavam aguardando atendimento na sala de espera, nos dias destinados à coleta dos dados.

Constituíram-se da amostra as mulheres que atenderam aos seguintes critérios de inclusão: ser cadastrada no serviço; estar no climatério, independente de haver menopausa; ter idade entre 35 e 65 anos; aceitar formlamente em participar do estudo, podendo desistir no momento em que achasse conveniente.

A coleta de dados foi realizada a partir de entrevistas semiestruturadas, seguindo um roteiro com perguntas fechadas contendo os dados de identificação e perguntas abertas relacionadas à temática, tais como o conhecimento da mulher sobre o climatério/menopausa, os sintomas presentes durante $o$ climatério, as modificações ocorridas nessa fase, o tratamento utilizado, dentre outros necessários para o alcance dos objetivos.

Os resultados foram tabulados no programa EPI INFO, e organizados em tabelas, que foram interpretados e em seguida fundamentados na literatura pertinente à temática.

Os aspectos éticos e legais seguiram os critérios sobre pesquisa envolvendo seres humanos, estabelecidos na Resolução 196/96 ${ }^{(10)}$. Para tanto, o projeto foi aprovado pelo Comitê de Ética e Pesquisa da Universidade de Fortaleza, sob protocolo n. 045/2006.

\section{RESULTADOS}

Na presente pesquisa, foram entrevistadas 101 mulheres que atenderam os critérios de inclusão e que discorreram sobre a sintomatologia do climatério. Para proceder à análise e à discussão dos resultados optouse por tabular os dados em quadros para uma melhor visualização.

Para caracterizar os aspectos sociodemográficos das mulheres entrevistadas, no período do climatério, ressaltamos que a idade delas variou de 35 a 68 anos, tendo como média de idade 53 anos. Este grupo de mulheres foi composto, em sua maioria, por mulheres brancas, correspondendo a 64(63,36\%), enquanto que $37(36,64 \%)$ eram consideradas da categoria nãobranca. Das mulheres entrevistadas 7(6,93\%) moravam sozinhas e 94(93,07\%) moravam acompanhadas. Todas eram procedentes de Fortaleza.

Quanto ao nível educacional os resultados foram bem variados, porém satisfatório, uma vez que apenas 3 das pacientes eram analfabetas. Quando questionadas sobre a ocupação que tinham, as respostas obtidas foram: 61(60,4\%) não tinham uma ocupação, $11(10,89 \%)$ trabalhavam com serviços gerais, $3(2,97 \%)$ eram autônomas, 8(7,92\%) eram aposentadas e 18(17,82\%) tinham outras ocupações (costureira, professora, manicure, auxiliar de enfermagem, auxiliar de dentista, auxiliar de escritório e telefonista).

O grupo de mulheres entrevistadas, diante dos critérios de inclusão, deveria obrigatoriamente estar vivendo o período do climatério, porém seria independente da presença de menopausa, uma vez que o climatério tem início por volta dos 35 anos e a menopausa ocorre aproximadamente aos 50 anos. Portanto, demonstra-se com a tabela 1 a presença ou não do

Tabela 1 - Divisão de mulheres quanto à presença de menopausa. Fortaleza, 2009

\begin{tabular}{lcc}
\hline & Presença de menopausa & n \\
\hline Sim & 73 \\
Não & 28 \\
\hline Total & 101 \\
\hline
\end{tabular}

climatério nas mulheres entrevistadas.

Como resultado foram obtidos, no grupo entrevistado, que 73(72,28\%) mulheres já se encontrava na menopausa, enquanto $28(27,72 \%)$ estavam no período pré e perimenopausa.

A média de idade em que foi observada a menopausa foi de 47 anos, havendo uma variação de 25 a 58 anos. É importante salientar que a variação de menopausa espontânea foi de 39 a 58 anos, destacando-se que duas mulheres haviam sido submetidas à histerectomia total e parcial, com idades de 25 e 30 anos, respectivamente.

Na tabela 2 caracteriza-se a presença ou não de sintomas do climatério nas mulheres entrevistadas.

A sintomatologia associada à síndrome do climatério foi altamente prevalente, sendo que cerca de $93(92,07 \%)$ mulheres experimentaram pelo menos um dos sintomas, contra $8(7,93 \%)$ que não relataram sintomatologia.

Tabela 2 - Divisão de mulheres no climatério quanto à presença sintomas. Fortaleza, 2009

\begin{tabular}{lcc}
\hline Presença de sintomas & $\mathbf{n}$ & $\mathbf{\%}$ \\
\hline Sim & 93 & 92.07 \\
Não & 08 & 7.93 \\
\hline Total & 101 & 100 \\
\hline
\end{tabular}


A prevalência dos sintomas na menopausa é demonstrado na tabela 3, o qual encontra-se a presença de sintomas como: fogachos, irritabilidade, esquecimento, dores nas pernas, cefaleia, diminuição da libido, insônia e depressão, atrofia vaginal e outros. Outros sintomas mencionados incluem tonturas, ansiedade, palpitações, sudorese intensa, incontinência urinária, fadiga e fluxo menstrual intenso.

Tabela 3 - Prevalência dos sintomas na menopausa. Fortaleza, 2009

\begin{tabular}{ll}
\hline Sintomas na menopausa & fa \\
\hline Fogachos & 82 \\
Irritabilidade & 79 \\
Esquecimento & 72 \\
Dores nas pernas & 68 \\
Cefaléia & 58 \\
Diminuição da libido & 55 \\
Insônia e depressão & 51 \\
Atrofia Vaginal & 44 \\
Outros Sintomas & 44 \\
\hline
\end{tabular}

Cerca de dois terços das mulheres procuraram o serviço médico devido aos sintomas climatéricos, sendo que a maior causa relacionada são os sintomas vasomotores seguidos dos psicológicos. Apresentase, portanto, na tabela 4, a distribuição das mulheres quanto a presença de sintomas, assistência médica e realização de Terapia de Reposição Hormonal (TRH).

Tabela 4 - Distribuição de mulheres no climatério quanto à presença de sintomas, assistência médica e realização de Terapia de Reposição Hormonal (TRH). Fortaleza, 2009

\begin{tabular}{lcc}
\hline Características & Sim & Não \\
\hline Sintomas & 93 & 08 \\
Assistência médica & 73 & 28 \\
TRH & 29 & 73 \\
\hline
\end{tabular}

Dentre as causas que levaram essas mulheres à busca pelo serviço médico especializado foram mencionados a sintomatologia intensa $(49,50 \%)$, encaminhamento de outro especialista $(34,65 \%)$ e outras causas $(1,98 \%)$.

\section{DISCUSSÃO}

A mulher é prioridade nas Políticas Públicas de Saúde, mas apenas por ser reprodutiva; ao entrar no climatério perde, em grande parte, essa atenção do Serviço de Saúde. Portanto, a enfermeira, por contactar em mais oportunidades com a mulher, como no caso do Programa de Tratamento do Câncer Ginecológico (20-59 anos), tem mais condições de auxiliá-la em todas as etapas da vida. Nesse sentido, são fundamentais as iniciativas que contemplem a atenção à saúde, incluindo a possibilidade de troca de experiências, acesso à informações em uma assistência holística, para que a mulher no climatério alcance a autovalorização e a auto-estima, fundamentais ao bem-estar e à longevidade com saúde e dignidade ${ }^{(11)}$.

O climatério é um período considerado de etiopatogenia complexa; embora envolva todo o eixo hipotálamo-hipófise-ovário, sendo o ovário a estrutura mais relevante nesse processo. Nele ocorre, progressivamente, a diminuição dos folículos e os remanescentes tornam-se refratários às gonadotrofinas. Esta diminuição leva ao declínio progressivo dos estrogênios e observa-se elevação progressiva das gonadotrofinas, na tentativa de manter a foliculogênese, as quais, atuando sobre o estroma do ovário, fazem com que haja maior produção de androgênios. Assim, algumas mulheres conseguem o equilíbrio endócrino, principalmente, as que têm boa alimentação desde a infância, praticam esportes e estão bem realizadas sob o ponto de vista emocional e social ${ }^{(12)}$.

Estudiosos corroboram com essa assertiva quando ressalta que fatores genéticos, doenças e cirurgias, nuliparidade, dieta vegetariana, grandes altitudes, magreza, uso de medicamentos quimioterápicos e hábito de fumar podem influenciar a ocorrência do climatério. Afirmam ainda que a multiparidade, o aumento da massa corpórea e até mesmo a história de melhor desempenho intelectual na infância podem interferir na idade em que ocorre a menopausa, prolongando o período de produção estrogênica. Entretanto, não existem estudos suficientes que afirmem que o uso de contraceptivos orais, a idade da menarca, a idade da menopausa da mãe, o nível socioeconômico e social e a raça podem ter relação com a ocorrência de menopausa, precoce ou $\operatorname{tardia}^{(13)}$.

Pode-se observar, posteriormente, o surgimento dos sintomas vasomotores, dentre eles: fogachos (ondas de calor, descritos como uma sensação de pressão na cabeça, seguido pela sensação de calor na cabeça, pescoço e tórax) e a taquicardia, referidos como palpitações. Os sintomas psiquiátricos, como a depressão, normalmente vêm a seguir aos vasomotores. E, como sintomas mais tardios, observam-se alterações meta- 
bólicas, osteoporose, doença cardiovascular e doença de Alzheimer ${ }^{(13-14)}$.

As mulheres com maior risco de ter fogachos são aquelas na pós-menopausa, com 50-54 anos de idade, não brancas, vivendo com companheiro, pertencentes aos estratos mais pobres da população e com índice de massa corpórea compatível com sobrepeso e obesida$\mathrm{de}^{(15)}$. O uso de métodos anticoncepcionais hormonais, após os quarenta anos de idade, apresenta efeito protetor sobre a ocorrência de fogachos.

Quando questionadas sobre a libido, muitas mulheres respondiam negativamente, porém com o desenvolver da conversa, elas sentiram-se mais à vontade para dar os esclarecimentos, muitas vezes alterando a resposta dada anteriormente, referindo constrangimento. A perda da libido é um sintoma comum no climatério, pode estar relacionado com fatores hormonais, principalmente androgênicos, e psicológicos. Porém os resultados da pesquisa mostraram a diminuição do interesse por sexo mais prevalente do que a atrofia vaginal, no entanto todas as mulheres que referiram à vaginite atrófica revelaram simultaneamente a diminuição da libido.

A atrofia vaginal ocorre mais comumente quatro a seis anos após a menopausa e está diretamente ligada aos níveis reduzidos de estrogênio. Foi comprovado que as mulheres que têm parceiro sexual apresentam mais queixas genitais do que as que não têm parceiro, uma vez que com a diminuição da atividade sexual poderia reduzir a percepção da atrofia vaginal ${ }^{(9)}$.

Dentre a população estudada, nenhuma apresentou diagnóstico médico de depressão, porém 51 das pacientes informaram sentir-se deprimidas. Portanto, a depressão aqui evidenciada não está direcionada ao diagnóstico médico, mas àquela conhecida pelo senso comum, quando verbalizada pelas próprias pacientes. O climatério é uma fase de transição, envolve mudanças hormonais que interferem nos sentimentos e respostas físicas, psicológicas e sociais, é um momento de considerável estresse e, portanto, de risco para o desenvolvimento de depressão ${ }^{(2)}$.

A insônia é frequentemente atribuída às ondas de calor, mas também pode estar associada aos sintomas psicológicos, o que refletiria a sua associação com depressão ${ }^{(16)}$. No presente estudo, a insônia relacionouse tanto com os sintomas vasomotores como com os psicológicos, embora tenha sido observada uma associação mais íntima com os sintomas vasomotores, provavelmente, decorrente da sintomatologia clássica: fogachos e sudorese noturnos gerando a insônia e, em consequência, irritabilidade e fadiga no dia posterior.

Algumas mulheres que apresentavam sintomas referiram não realizar um acompanhamento devido à dificuldade de acesso às unidades de saúde e de agendamento de consultas com o especialista. Outras referiram não achar necessário esse tipo de acompanhamento, por acreditar que a sintomatologia seria passageira. As mulheres que não apresentavam sintomas referiram não precisar de acompanhamento do climatério. A principal causa pela qual as mulheres no climatério não procuram atendimento médico é a consideração de que os sintomas não mereciam atenção médica por serem naturais, ressaltando também, a dificuldade de acesso a serviços de saúde para a nãoprocura por atenção médica.

Com relação à terapia de reposição hormonal (TRH), 28,72\% das mulheres que realizavam mostraram-se satisfeitas, referindo alívio considerável dos sintomas, porém queixavam-se de não existir um programa de distribuição gratuita dos hormônios. Algumas mulheres que não realizavam TRH disseram não aderir ao tratamento devido ao custo elevado, medo de efeitos adversos, dentre eles câncer de mama e falta de informação sobre o tratamento. Outras pacientes iniciaram o tratamento com hormônio, porém não tinham condições financeiras de dar continuidade, ou por falta de conhecimento desistiram da terapia.

A prevalência de sintomas no climatério, como fogachos, sudorese, palpitação, tontura, ansiedade, irritabilidade, cefaleia, depressão e insônia, foi muito elevada na população estudada, confirmando-se que a transição do climatério tem um grande impacto para essas mulheres, ocasionando os transtornos em suas vidas, e assim dificultando o enfrentamento dessa fase.

Diante do presente estudo, infere-se que as políticas de atenção à saúde da mulher formuladas nacionalmente através de amplas e complexas discussões trouxeram contribuições imprescindíveis para o processo de transformação sobre o paradigma da saúde da mulher, em especial as que estão no período do climatério. Contudo, apesar dos avanços extraordinários, esse processo é dinâmico e acompanha a transformação da sociedade e, por isso, inexaurível. Portanto, a valorização da mulher, enquanto ser humano e cidadã, é algo destacável, e, por isso, os profissionais de enfermagem precisam atualizar-se e promover melhorias no cuidado de enfermagem à mulher no climatério ${ }^{(17)}$.

Ressalta-se, portanto, a importância da utilização de grupos como estratégia tecnológica para a promoção da saúde e em atividades do ensino, considerando, 
inclusive, as propostas oficiais do Ministério da Saúde e da Educação. Assim, o domínio dessa tecnologia pelos enfermeiros como fundamento para guiar suas ações assistenciais e gerenciais, torna-se a cada dia de maior importância. Enfatizando o equilíbrio para fazer intervenções sobre os conteúdos que emergem na dinâmica grupal, centradas no contexto científico e no emocional que surgem do contato com o grupo, em especial àqueles formado por mulheres no período do climatério ${ }^{(18)}$.

\section{CONCLUSÃO}

Conclui-se, diante do presente estudo, que o enfermeiro está em uma posição excelente, com suas interações, para ajudar a desmistificar as atitudes e as crenças da sociedade sobre essa etapa de vida de transição das mulheres, agindo como facilitadores do processo de significação e direcionamento da mulher. Portanto, como um educador em saúde e utilizando tecnologias educativas, os enfermeiros precisam encorajar as mulheres a visualizar a menopausa como uma alteração natural que resulta em libertar-se das menstruações e dos sintomas relacionados às alterações hormonais.

Portanto, cabe aos profissionais de enfermagem adotar medidas de acompanhamento, que visam prevenir as doenças e promover a saúde. O enfermeiro deve explicar à paciente que a cessação dos ciclos menstruais é uma função fisiológica, raramente acompanhada por sintomas ou doença nervosa que acometam modificações em seu cotidiano.

Diante disso, ressalta-se o importante e autônomo papel da enfermagem na interface com a saúde reprodutiva e na saúde coletiva, em especial com as mulheres no período do climatério, sendo relevante que elas se apropriem de fonte de informação sobre sua saúde e o manejo do climatério, afim de uma melhor qualidade de vida. Para tanto, o acolhimento aos usuários, nas unidades básicas de saúde, possibilita à enfermeira o primeiro contato com estas mulheres nas questões relativas à saúde da família como um todo.

\section{REFERÊNCIAS}

1. Lorenzi DRSD, Catan LB, Moreira K, Artico GR. Assistência à mulher climatérica: novos paradigmas. Rev Bras Enferm. 2009;62(2):287-93.

2. Almeida LHRB, Luz MHBA, Monteiro CFS. Ser mulher no climatério: uma análise compreensiva pela enfermagem. Rev Enferm UERJ. 2007;15(3):370-75.

3. Lorenzi DRS, Basso E, Fagundes PO. Prevalência de sobrepeso e obesidade no climatério. Rev Bras Ginecol Obstet. 2005;8(27):479-84.

4. Fonseca AM, Bagnoli VR, Cardoso EB. Climatério: abordagem atual do diagnóstico e tratamento. Rev Bras Med. 2004;1/2(61):65-9.

5. Freitas MF, Silva ARV, Silva RM. Mulheres vivenciando o climatério. Acta Scientiarum. Health Sciences. 2004;1(26):121-28.

6. Mori ME, Coelho VLD. Mulheres de corpo e alma: aspectos biopsicossociais da meia-idade feminina. Psicol Refl Crít. 2004;17(2):177-87.

7. Ramos LR, Rosa TE, Oliveira ZM, Medina MCG, Santos FRG. Perfil do idoso em área metropolitana na região sudeste do Brasil: resultados de inquérito domiciliar. Rev Saúde Pública 2007;27(2):87-94.

8. Arango GH. Bioestatística: teórica e computacional. $2^{\mathrm{a}}$ ed. Rio de Janeiro: Guanabara Koogan; 2005.

9. Oliveira DM, Jesus MCP, Merighi MAB. Climatério e sexualidade: a compreensão dessa interface por mulheres assistidas em grupo. Texto \& Contexto Enferm. 2008;17(3):519-26.

10. Ministério da Saúde (BR). Conselho Nacional de Saúde. Diretrizes e normas regulamentadoras de pesquisa envolvendo seres humanos. Resolução n. 196, de 10 de outubro de 1996. Brasília; 1996.

11. Berni NIO, Luz MH, Kohlrausch SC. Conhecimento, percepções e assistência à saúde da mulher no climatério. Rev Bras Enferm. 2007;60(3):299-306.

12. Fonseca AM, Bagnoli VR, Arie WMY. A dúvida do ginecologista: prescrever ou não hormônios na mulher no climatério? Rev Assoc Med Bras. 2009;55(5):507-507.

13. Gonçalves R. Vivenciando o climatério: o corpo em seu percurso existencial à luz da fenomenologia [tese]. São Paulo: Universidade de São Paulo; 2005.

14. Berek JS, Adashi EY, Hillar PA. Novak Tratado de ginecologia. 13 ${ }^{\mathrm{a}}$ ed. Rio de Janeiro: Guanabara Koogan; 2005.

15. Sclowitz IKT, Santos IS, Silveira MF. Prevalência e fatores associados a fogachos em mulheres climatéricas e pós-climatéricas. Cad Saúde Pública. 2005;2(21):469-81. 
16. Pedro AO, Pinto-Neto AM, Costa-Paiva LHS. Síndrome do climatério: inquérito populacional domiciliar em Campinas. Rev Saúde Pública. 2003;6(37):735-42.

17. Freitas GL, Vasconcelos CTM, Moura ERF, Pinheiro AKB. Discutindo a política de atenção à saúde da mulher no contexto da promoção da saúde. Rev Eletron Enferm. 2009;11(2):424-28.

18. Mota KAMB, Munari DB. Um olhar para a dinâmica do coordenador de grupos. Rev Eletron Enferm. 2006;8(1):150-61. 